\title{
CAE Analysis and Structure Optimization Design of Injection Mold for Charge Upper Cover
}

\author{
Mubarak Abdullah Ali Mohsen Alhadad, Quan Wang \\ National Local Joint Engineering Laboratory of Intelligent Manufacturing Oriented Automobile Die \& Mold, \\ Tianjin University of Technology and Education, Tianjin, China
}

\begin{abstract}
This paper focuses on the design and analysis of the plastic injection mold for production of the charger upper cover ,that is the most commonly used in small household appliances to get the battery power, the model of plastic charger upper cover was designed in UG environment.The shape and internal structure of the part are more complicated and have outer dimensions the wall thickness is uniform, the charge upper cover was analyzed under side gates design and compare in two different locations with cooling system for optimum deign .The first design is a double cavity mold with a single-gate and secondary runner system in the side and at the middle of charge upper cover, the second design is a double cavity mold with two gates and primary runner system, secondary runner system on the side of the product with the specific dimensions by mold flow that is possible to determine the plastic part elasticity and product quality, it can also anticipate possible defects and deflections in the model to improve mold structure to achieve low production costs and to get high quality and efficiency. So, by comparing the results of analysis such as warpages we found that a double cavity mold with two gates in the side of the product is better than a singlegate in the side at the middle of the product.
\end{abstract}

Keywords: CAE; Injection Molding; Optimization design; Charger upper cover.

\section{INTRODUCTION}

The injection mold process is the most commonly used process technology in manufacturing plastic especially nowadays the most popular method to produce 3dimensional parts of different kinds of polymeric materials. It is a suitable method for thermoplastics, thermosets, elastomers, short fiber, and particulate filled polymers; the injection molding process is an extremely used manufacturing process for plastic parts. The process is so versatile [1]. Injection molding is an engineering process that requires accurate planning and focuses on details of any product is to achieve its full potential, dimensional stability, defects free surface of products, excellent properties, less cost high efficiency, economy, and quality. It is a costeffective technique to produce small, complex-shaped, precision parts in enormous quantities [2]. The injection molding process consists of four important tasks: filling, packaging, cooling and ejection [3]. The most significant process parameters affecting the surface accuracy of products are melt temperature, injection pressure, packing pressure and packing time [4]. When manufacturing injection-molding parts, there are many factors affecting part's final cost such as part design, manufacture time, temperature and pressure used. The types of resin chosen plastic materials are generally used in all parts of the industry. The most important reason is the material properties of the plastic. Some of these properties are lightweight, corrosion resistance, and ease of getting shaped.
However, physical and chemical properties can be changed as desired [5]. The uses of injection-molded parts are numerous and growing with increasing demand from medical, automotive, industrial, household appliances, electronics covers of all equipment and many industries. Due to wide variety of plastics, the process performance and molding process of the materials are also very different. The selection of suitable plastic materials should be considered in terms of molding process characteristics, uses, and comprehensive performance. As growth continues, more manufacturers are replacing traditional materials such as wood, steel, and fiberglass with thermoplastic because of the physical and chemical properties of these materials. However, CAE has made a major impact on the design and manufacturing process in the injection industry in terms of both quality improved and cost reduction of applications of various computer simulation technique, however even more advanced techniques are demanded from this progressive industry. This paper is mainly for the injection mold design and mold flow analysis of the charger cover that is the most commonly used in small household appliances to obtain the battery power. In this paper, we design gates for multi-cavity injection molds for charger upper cover and cooling system and optimal warpage. The first design is the double cavity mold with a single-gate configuration while the second design is the double cavity mold with two gates configuration. The analysis results manifest that the double cavity mold with two gates configuration performs better than the double cavity mold with a single-gate configuration. The paper is arranged as follows: presents the design and molding process analysis then simulation analysis. Finally, conclusion.

\section{MOLDING PROCESS ANALYSIS}

\subsection{Comprehensive analysis of the upper charger cover}

This paper is mainly for the injection mold deign and mold flow analysis for charge upper cover which is an electric product .It should be taken into consideration to choose suitable plastic material in terms of molding properties ,uses and overall performance .The material PP has the best highfrequency insulation ,cheap, colorless, odorless, transparent, good in coloring, moldability, good in hardness ,low moisture absorption ,tensile strength, pressure strength .Polypropylene has a large surface resistance, does not absorb water , and has good heat resistance .Mainly used in electrical appliances[6].

\subsection{Design upper cover of the charger}

In this section we design injection mold design and model flow analysis of the charger cover. The cover is widely used 
in small household appliances battery power, as shown in Fig.1. The shape and internal structure of the part are relatively complex. Its overall dimension is $101.7 \mathrm{~mm} \times$ $49.9 \mathrm{~mm} \times 21.1 \mathrm{~mm}$, the wall thickness is basically uniform, the side wall thickness is $1.5 \mathrm{~mm}$, there are side holes, there are excessive fillets around, including some broken surfaces, which need to be repaired with mold tools. There are two side bulges on the inner walls of both sides of the part, and the size is $6.0 \mathrm{~mm} \times 2.0 \mathrm{~mm} \times 1.0 \mathrm{~mm}$, which need to be designed with side core pulling mechanism. The product demand is large, the appearance is beautiful and the quality is reliable.

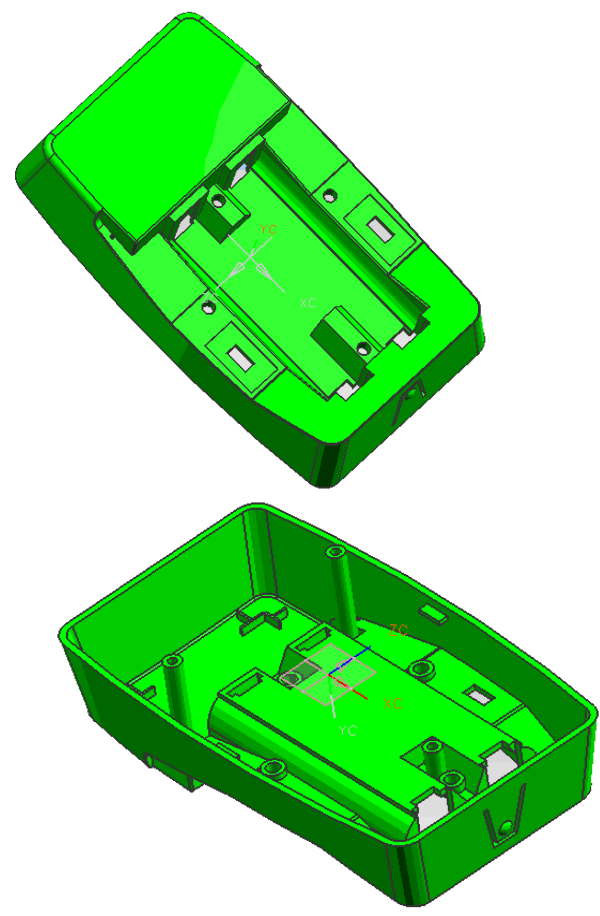

Fig. 1 Structure of charger upper cover

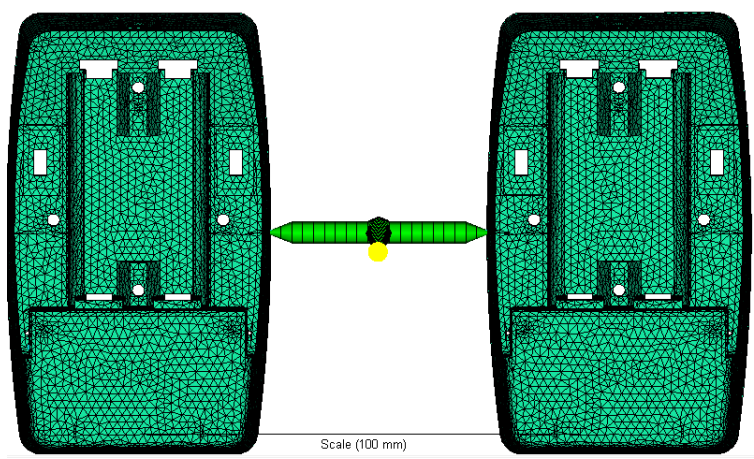

Fig 2(a). Double cavity mold with a single-gate

\section{DESIGN OF COOLING SYSTEM}

The cooling stage is the most important stem amongst in injection mold [12]. It has critical role during the injection molding process [13] it determines the rate at which parts

\section{DESIGN OF GATES}

According to plastic injection mold design, the design of the runner and gating systems is the most important to realize a successful injection molding process [7]. The position of the injection gate is of great importance since it can affect the flow direction and melt solidification through and after filling [8]. Gate position is among the most critical factors in the achieving dimensionally accurate parts and high productivity of the molding process [9]. The gate is one of the most important parts in mold flow is where and how the gate must be located, as the size, shape, and location of the gate are important factors in how to build everything. It should determine the cavity layout of the charger cover according to the product requirements and the experiments, the beginning the design process adopted double cavity mold with a single-gate in the side at the middle of product and double cavity mold with two gates on the side of product with the specific dimensions, as shown in Figure2. Both products are under the same influential conditions complete analysis is done from the structural shape size, and thickness of wall of the product, and the side gates are selected and placed on the parting surface, to feed on the side of the plastic part. It is simple to remove the waste created by the casting system without influencing the appearance of the plastic part. The gate area is small, the melt is pressed and sheared before entering the cavity, the flow condition is improved, the molding is easy, the surface smoothness of the product is improved. The

residual stress in the vicinity becomes small prevent deformation, cracking backflow. The cold sprue is tapered circular shape. That has dimensions the start diameter is $6 \mathrm{~mm}$ and end diameter is $2 \mathrm{~mm}$. Secondary runner's diameter system is $5 \mathrm{~mm}$, the cold gate's start diameter is $5 \mathrm{~mm}$ and the end diameter is $1 \mathrm{~mm}$. while the second design of side gates have is tapered circular shape that has dimensions the start diameter is $6 \mathrm{~mm}$ and the end diameter $2 \mathrm{~mm}$, primary runner's diameter system is $5 \mathrm{~mm}$, secondary runner's diameter system is $5 \mathrm{~mm}$.the cold gate's start diameter is $5 \mathrm{~mm}$ and the end diameter is $1 \mathrm{~mm}$. As shown in Figure 2.

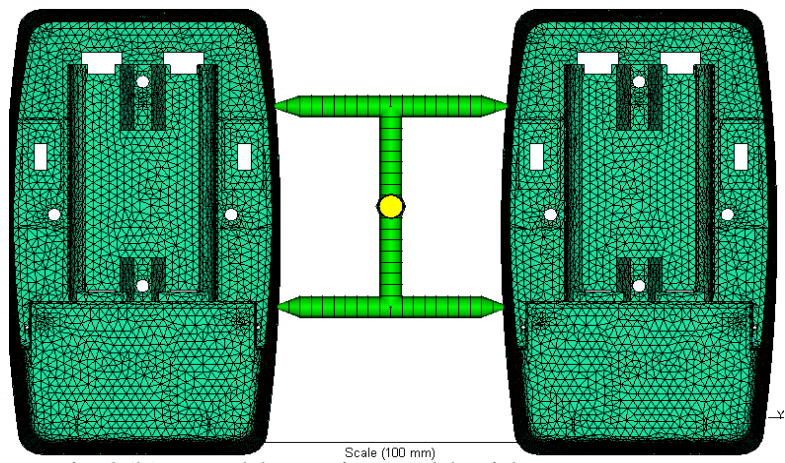

Fig 2(b). Double cavity mold with two gates

are produced, the quality of the cooling always affects the surface quality, crystallinity and leftover stress of the product. The generated effect by cooling system effectively reduces the molding cycle, improve product quality and 
production efficiency, and reduce production costs [14], also the warp can be reduced, and to control of the formation of frozen layer and warpage, the appreciate selection of injection time melt temperatures [10], packing pressure [11], and packing time would be required. The design of the cooling system is such that the surface temperature of the mold is closed and the plastic part are cooled evenly, the cooling water passage should be as close as possible to the surface of the cavity. The distance from the cooling water hole to the surface of the cavity, is greater than $10 \mathrm{~mm}$. The proximity of the cooling passage to the molding portion, that is, the size of the heat conduction area and the length of the heat conduction distance, cooling channel length and arrangement the size of the cooling passage and the flow state of the cooling medium. Temperature difference between inlet and outlet coolant $\left(5^{\circ} \mathrm{C}\right.$ is appropriate).

Moldflow automatically creates a cooling system. The cooling system based on the cooling ring processor is shown in Fig .3. In the original cooling system, the cooling pipe is divided into two upper and lower layers, each using 4 tubes with a diameter of $8 \mathrm{~mm}$, which is connected by a plastic hose, and water fed from the left side of the pipe. Distance between channels center is $30 \mathrm{~mm}$, the distance between two cooling channels is $20 \mathrm{~mm}$, and the distance to extend beyond part is $30 \mathrm{~mm}$. using cooling water as a cooling medium, the inlet temperature is $25^{\circ} \mathrm{C}$.

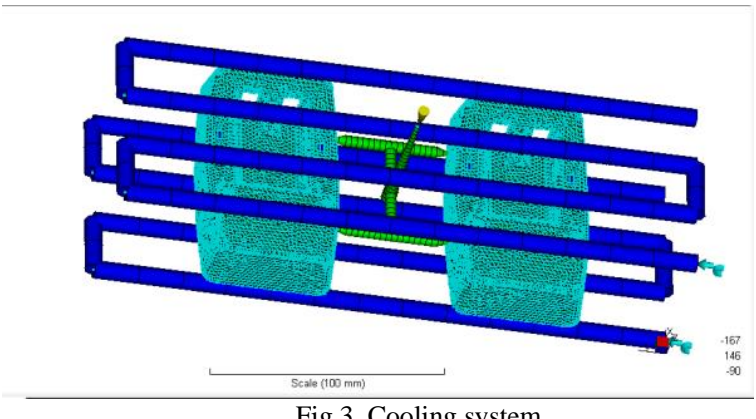

\section{SIMULATION ANALYSIS}

\subsection{Warpage analysis}

The warpage for a single -gate and two gates shown in Fig4 the maximum warpage of a single-gate configuration is $0.5830 \mathrm{~mm}$ shown in Fig 4(a) and the maximum warpage of two gates configuration is $0.4651 \mathrm{~mm}$ as shown in Fig 4(b) which are less than the single-gate warpage. Note that the single-gate design exceeded the allowable limit according to customer standards and allowed warpage should be less than $5 \mathrm{~mm}$, during the results of analysis it is clear that the two gates design system configuration is better than the singlegate design system configuration.

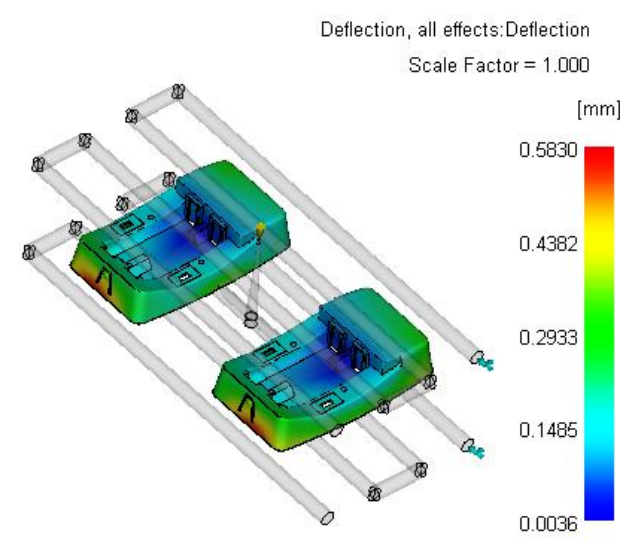

Fig4(a). Warpage for a single gate

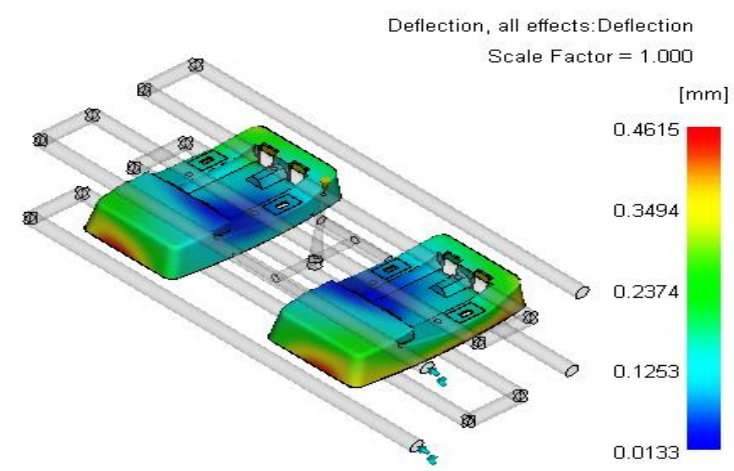

Fig 4(b). Warpage for two gates

\subsection{Air traps}

Air traps are dependent mostly on the gating scheme design and geometry of the parts [15], certainly, it is a part defect that shows us a little bubble on the surface of the part and it generally formed by improper or cooling off by gas or air bubbles. That have formed inside that plastic, the result will be an imperfect product this Fig. 5 show the distribution of bubbles on the part. This product has basic air holes on the surface of the charger cover so the gas can be discharge from the cavity during the injection process.

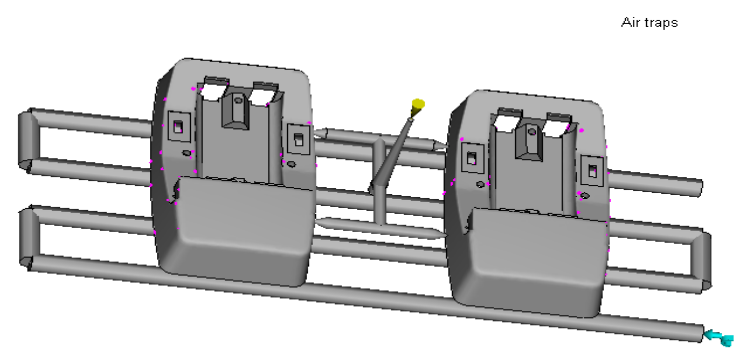

Fig5. Air traps 


\subsection{Weld lines}

Weld lines in any a molded part by injection system, they are critical defects. The weld lines cannot be avoided when designing multiple gates in a molding, they should be positioned in the non-critical region [16].The structure of plastic parts should be reasonable and the thickness is moderate .The wall thickness should be even ,As shown in Fig.6 the result of the weld line superimposed on the fill time, the position at the last thin line of the weld mark is not clear on the outer part of the surface, there are stiffeners in the parts. Which will not affect the appearance and strength.

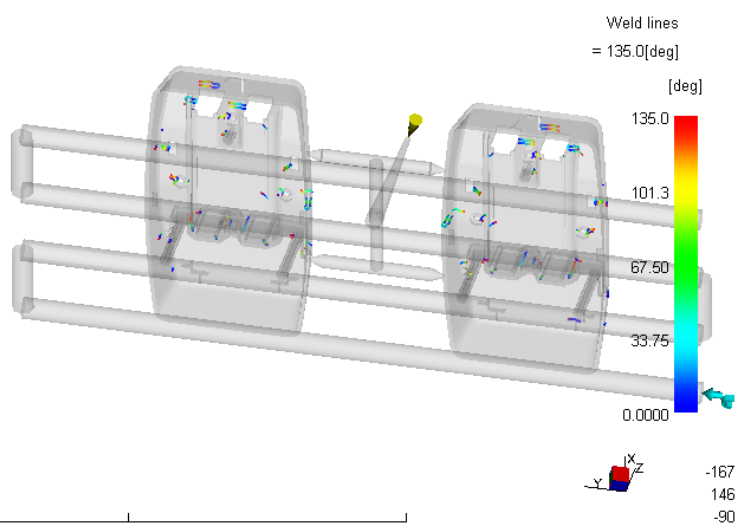

Fig. 6 Weld lines

\section{CONCLUSION}

A design and optimization the gates location, cooling system and process parameters are designed Autodesk Mold flow 2012 to simulates the filling of the charger upper cover .Through the analysis of both designs we obtained several results, the most important of these result is the warpages as shown in the Fig4(a,b) that double cavity mold with two gates on the side has less warpages than the double cavity with the single-gate of product. We have just improved the flow channel injection system and cooling system used the mold flow to simulates the filling of the charger upper cover so it is reasonable to get deformations, weld lines, and other defects those absolutely effect on the quality of product .This is not comprehensive analysis so we suggest that in the future determine the shape and layout of the mold through the location of the gates, cooling system passages design and parameters of the molding process, should choose the melt temperature, injection pressure and injection time to ensure lower deformations, void defects and improve product.

\section{REFERENCES}

[1] Mircheski, I., Łukaszewicz, A., \& Szczebiot, R. (2019). Injection process design for manufacturing of bicycle plastic bottle holder using CAx tools. Procedia Manufacturing, 32, 68-73

[2] Qin, M., Lu, H., Wu, H., He, Q., Liu, C., Mu, X., ... \& Qu, X. (2019). Powder injection molding of complex-shaped aluminium nitride ceramic with high thermal conductivity. Journal of the European Ceramic Society, 39(4), 952-956.
[3] Khosravani, M. R., \& Nasiri, S. (2019). Injection molding manufacturing process: review of case-based reasoning applications. Journal of Intelligent Manufacturing, 1-18.

[4] Bensingh, R. J., Machavaram, R., Boopathy, S. R., \& Jebaraj, C. (2019). Injection molding process optimization of a bi-aspheric lens using hybrid artificial neural networks (ANNs) and particle swarm optimization (PSO). Measurement, 134, 359-374.

[5] Ozcelik, Babur, and Ibrahim Sonat. "Warpage and structural analysis of thin shell plastic in the plastic injection molding." Materials \& Design 30.2 (2009): 367-375.

[6] Karger-Kocsis, J. (Ed.). (2012). Polypropylene: an AZ reference (Vol. 2). Springer Science \& Business Media.

[7] Lee, K. S., \& Lin, J. C. (2006). Design of the runner and gating system parameters for a multi-cavity injection mould using FEM and neural network. The International Journal of Advanced Manufacturing Technology, 27(11-12), 1089-1096.

[8] Huszar, M., Belblidia, F., Davies, H. M., Arnold, C., Bould, D., \& Sienz, J. (2015). Sustainable injection moulding: The impact of materials selection and gate location on part warpage and injection pressure. Sustainable Materials and Technologies, 5, 1-8.

[9] Hassan, H., Regnier, N., \& Defaye, G. (2009). A 3D study on the effect of gate location on the cooling of polymer by injection molding. International Journal of Heat and Fluid Flow, 30(6), 12181229.

[10] Song, M. C., Liu, Z., Wang, M. J., Yu, T. M., \& Zhao, D. Y. (2007) Research on effects of injection process parameters on the molding process for ultra-thin wall plastic parts. Journal of Materials Processing Technology, 187, 668-671.

[11] Tang, S. H., Tan, Y. J., Sapuan, S. M., Sulaiman, S., Ismail, N., \& Samin, R. (2007). The use of Taguchi method in the design of plastic injection mould for reducing warpage. Journal of Materials Processing Technology, 182(1-3), 418-426.

[12] Shinde, M. S., \& Ashtankar, K. M. (2018). Effect of different shapes of conformal cooling channel on the parameters of injection molding. Computers Materials \& Continua, 55(1), 287-306.

[13] Tang, Y., Gao, Z., \& Zhao, Y. F. (2019). Design of conformal porous structures for the cooling system of an injection mold fabricated by Additive Manufacturing Process. Journal of Mechanical Design, 141(10), 101702.

[14] Park, H. S., \& Dang, X. P. (2010). Optimization of conformal cooling channels with array of baffles for plastic injection mold. International Journal of Precision Engineering and Manufacturing, 11(6), 879-890.

[15] Shi, F., Lou, Z. L., Zhang, Y. Q., \& Lu, J. G. (2003). Optimisation of plastic injection moulding process with soft computing. The International Journal of Advanced Manufacturing Technology, 21(9), 656-661.

[16] Zhai, M., Lam, Y. C., \& Au, C. K. (2006). Runner sizing and weld line positioning for plastics injection moulding with multiple gates. Engineering with Computers, 21(3), 218-224. 